\title{
Long-term increase in coherence between the basal ganglia and motor cortex after asphyxial cardiac arrest and resuscitation in developing rats
}

\author{
Bhooma R. Aravamuthan ${ }^{1}$ and Michael Shoykhet ${ }^{1,2}$
}

BACKGROUND: The basal ganglia are vulnerable to injury during cardiac arrest. Movement disorders are a common morbidity in survivors. Yet, neuronal motor network changes postarrest remain poorly understood.

METHODS: We compared function of the motor network in adult rats that, during postnatal week 3, underwent $9.5 \mathrm{~min}$ of asphyxial cardiac arrest $(n=9)$ or sham intervention $(n=8)$. Six months after injury, we simultaneously recorded local field potentials (LFP) from the primary motor cortex (MCX) and single neuron firing and LFP from the rat entopeduncular nucleus (EPN), which corresponds to the primate globus pallidus pars interna. Data were analyzed for firing rates, power, and coherence between MCx and EPN spike and LFP activity.

RESULTS: Cardiac arrest survivors display chronic motor deficits. EPN firing rate is lower in cardiac arrest survivors (19.5 \pm 2.4 $\mathrm{Hz})$ compared with controls $(27.4 \pm 2.7 \mathrm{~Hz} ; P<0.05)$. Cardiac arrest survivors also demonstrate greater coherence between EPN single neurons and MCx LFP $(3-100 \mathrm{~Hz} ; P<0.001)$.

CONCLUSIONS: This increased coherence indicates abnormal synchrony in the neuronal motor network after cardiac arrest. Increased motor network synchrony is thought to be antikinetic in primary movement disorders. Characterization of motor network synchrony after cardiac arrest may help guide management of post-hypoxic movement disorders.

C ardiac arrest affects almost 20,000 children per year in the United States $(1,2)$. Long-term morbidity in survivors largely depends on post-arrest neurologic function $(2,3)$. Multiple brain structures are affected by the global hypoxia and ischemia that occurs with cardiac arrest, but the basal ganglia are particularly vulnerable (3-5). Consequently, postarrest movement disorders are common and often medically intractable $(4,6)$.

The basal ganglia-thalamo-cortical network is a modulatory conduit for motor information $(7,8)$. The striatum and subthalamic nucleus (STN) receive cortical input to the basal ganglia. The internal segment of the globus pallidus (GPi) and the substantia nigra pars reticulata send basal ganglia output to the thalamus. Dopamine signaling gates striatal transmission of cortical input to the GPi and substantia nigra pars reticulata which can occur directly or indirectly via the external segment of the globus pallidus and the STN. The GPi and substantia nigra pars reticulata ultimately transmit this modulated motor information back to the cortex via the motor thalamus $(7,8)$.

Electrophysiological changes in the basal ganglia have been extensively studied in multiple movement disorders, including Parkinson's disease and primary dystonia. The normal basal ganglia rarely display oscillatory firing and most structures fire irregularly (8). In Parkinson's disease, abnormally synchronous $\beta$ frequency activity $(12-35 \mathrm{~Hz})$ occurs within and between the GPi, STN, and the primary motor cortex (MCx) $(7,8)$. Beta frequency activity decreases with dopaminergic therapy or with voluntary movement and increases with voluntary inhibition of movement, which suggests that excessive $\beta$ frequency neuronal activity could be associated with abnormal movement patterns (7).

Synchronous activity in the lower frequency ranges $(\theta$ and $\alpha, 3-12 \mathrm{~Hz}$ ) may also be associated with abnormal movement, since it exists in the GPi in dystonia and correlates with dystonic EMG activity (9-12). In contrast, neuronal activity in the GPi in the $\gamma$ frequency range (35-100 Hz, primarily $60-90$ $\mathrm{Hz}$ ) increases with voluntary movement in Parkinson's disease and dystonia (13) and could be a signature of normal movement patterns. Abnormal movements in these diseases could involve hypokinesis (as with bradkyinesia in Parkinson's disease) or hyperkinesis (as with parkinsonian tremor or dystonic spasms). Frequency bands associated with abnormal movements (hypo- or hyperkinesis) are called antikinetic, while frequency bands associated with normal movement are called prokinetic.

Animal models of cardiac arrest have allowed for the examination of electrophysiological changes in the brain after global hypoxic-ischemic injury. Neurons in the thalamic ventral posteromedial nucleus show increased firing rates acutely and chronically after cardiac arrest, reflecting decreased intrathalamic inhibition from the injured thalamic reticular nucleus (14). In addition to modulating intrathalamic activity, the 
thalamic reticular nucleus is also poised to regulate transmission of oscillatory activity between basal ganglia output nuclei and the cerebral cortex (15). Abnormal synchrony in the basal ganglia-thalamo-cortical circuit would likely impair movement in survivors of cardiac arrest, yet post-arrest changes in this circuit remain poorly characterized. In the present study, we examined long-term alterations in coherence between the rat homologue of GPi, the entopeduncular nucleus (EPN), and the MCx in adult rats that survived asphyxial cardiac arrest and resuscitation during development.

\section{RESULTS}

We analyzed EPN single units, EPN local field potentials (LFPs), and MCx LFPs in rats 6-9 mo after they underwent either cardiac arrest ( $n=9$ rats, $87 \mathrm{EPN}$ units) or a sham intervention ( $n=8,92 \mathrm{EPN}$ single units) on postnatal day (P) $17-19$. We also examined motor function in a separate cohort of rats after cardiac arrest $(n=13)$ or sham intervention $(n=$ 11). Therefore, these results reflect chronic changes within and between the EPN and the MCx in adult rat survivors of cardiac arrest during development.

\section{EPN Single Unit Activity After Cardiac Arrest}

Firing rates of EPN neurons are 30\% lower in rats after cardiac arrest compared with sham rats $(19.5 \pm 2.4$ vs. $27.4 \pm 2.7 \mathrm{~Hz}$; t-test; $P<0.05$; Figure 1). Under our recording conditions, firing rates of EPN neurons in sham animals are similar to those reported previously in awake rats $(29.0 \pm 1.6 \mathrm{~Hz})(16)$ and freely moving rats $(26.0 \pm 3.1 \mathrm{~Hz})(17)$. Raw EPN spike power is lower in rats after cardiac arrest compared with sham animals across all frequency bands (two-way ANOVA; $P<0.01$; Figure 1), but there is no difference between groups when EPN spike power is normalized to total EPN spike power across all frequencies (two-way ANOVA; $P=0.12$; Figure 1). Since EPN spike power depends on EPN firing rate (see Methods), decreased power in rats after cardiac arrest compared with sham animals likely reflects the lower firing rates of EPN neurons in cardiac arrest survivors.

\section{Coherence Between EPN Spike, EPN LFP, and MCx LFP After Cardiac Arrest}

Despite decreased EPN firing rates, coherence between EPN spike activity and MCx LFP significantly increased after cardiac arrest. In sham rats, coherence between EPN spike and MCx LFP was significant only in very low frequency ranges (less than $3 \mathrm{~Hz}$ ). In rats after cardiac arrest, coherence became significant across all frequency bands (ANOVA; student's $t$-test; $P<0.001)$. Coherence in the $20-22 \mathrm{~Hz}$ frequency range, at the threshold between the low and high $\beta$ bands, is notably outside of the $95 \%$ confidence interval $(P<0.05$; Figure 2$)$. These data indicate increased synchrony between the EPN and MCx chronically after cardiac arrest, particularly in the antikinetic $\beta$ frequency range.

EPN spike activity and EPN LFP were not significantly coherent with each other, a relationship that remained unchanged after cardiac arrest (Figure 2). This is similar to what has been previously described in the analogous primate GPi $(18,19)$. In contrast, EPN LFP and MCx LFP demonstrate significant coherence in sham rats (Figure 2). After cardiac arrest, coherence between EPN and MCx LFPs is significantly decreased (ANOVA; $P<0.0001$; Figure 2).

\section{EPN LFP Power and MCx LFP Power Remain Unchanged After Cardiac Arrest}

There is a trend toward decreased EPN LFP power, especially in the $\theta$ and $\alpha$ frequency bands, in rats after cardiac arrest (Figure 3). Although this trend is still present after normalization to total power across the $0-100 \mathrm{~Hz}$ range (Figure 3), it does not reach statistical significance. MCx LFP power also shows no significant difference between rats after sham arrest
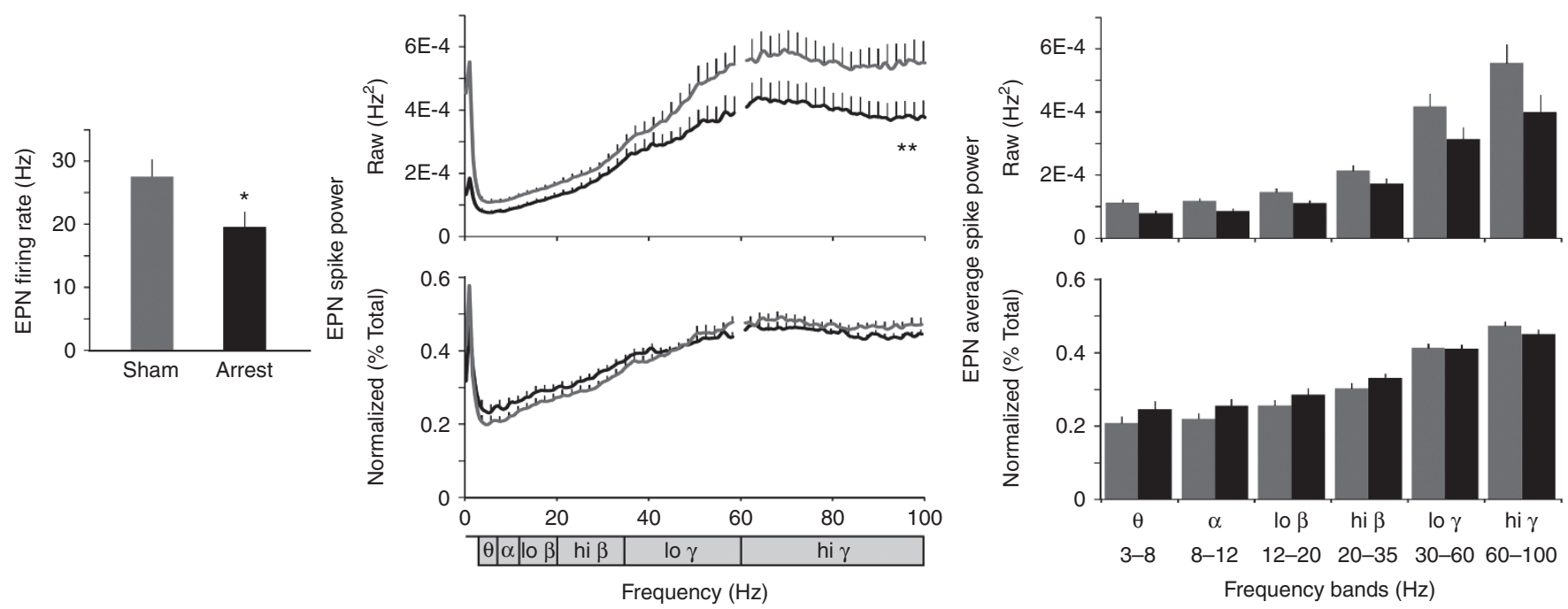

Figure 1. EPN spike characteristics after cardiac arrest and sham arrest. EPN firing rate (left), spike power spectra across $0-100 \mathrm{~Hz}$ (middle), and average spike power across frequency bands (right) are shown with SE (vertical error bars). Raw power (top row) was normalized (bottom row) by dividing power spectra by total power across $0-100 \mathrm{~Hz}$. Asterisks indicate significant differences in rate or power between cardiac arrest (black) and sham arrest (gray): ${ }^{* *}$ ANOVA to compare spectra, ${ }^{*}$ Student's $t$-test with Bonferroni correction to compare average power, $P<0.05$. EPN, entopeduncular nucleus. 

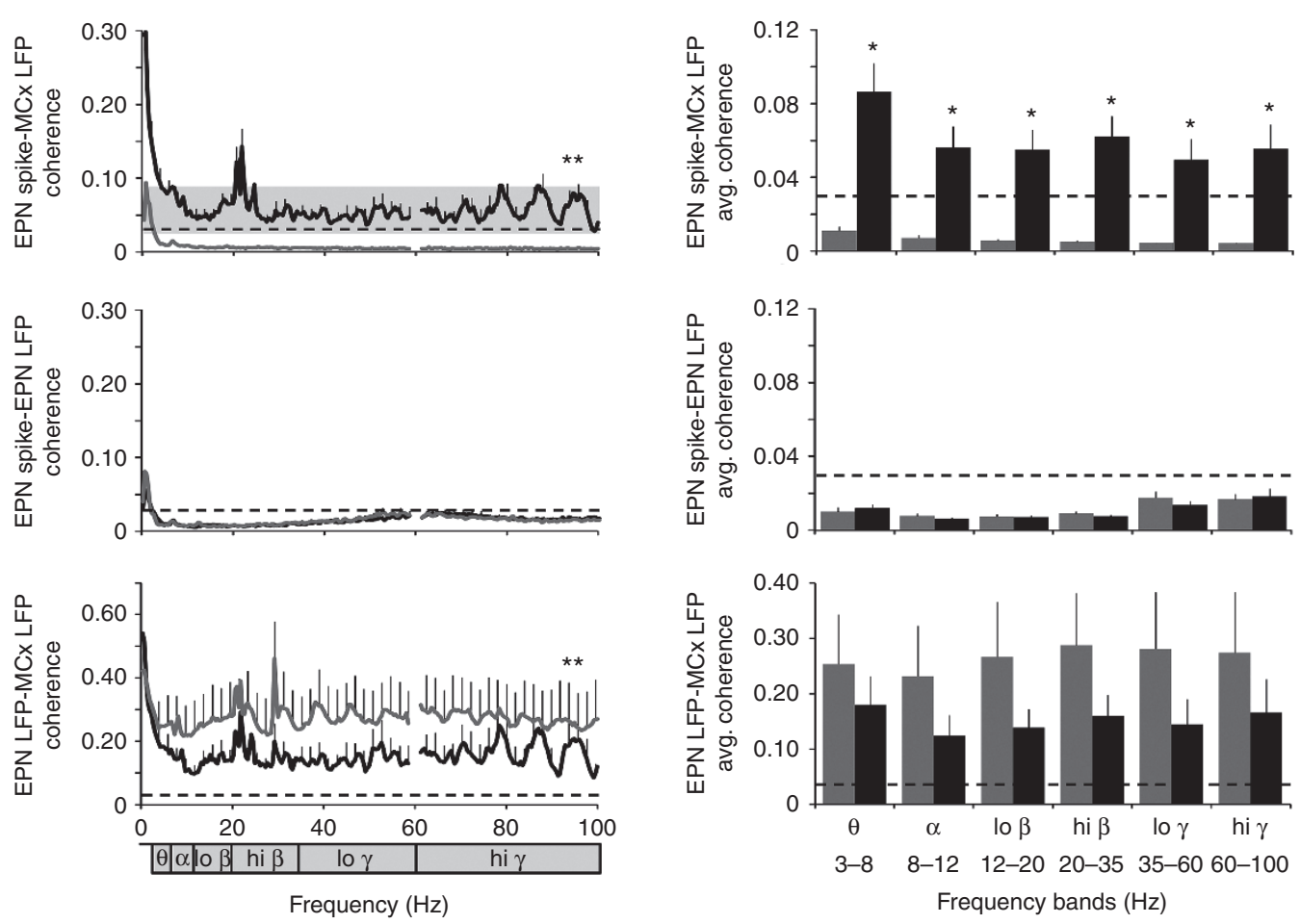

Figure 2. EPN spike and EPN-MCX LFP coherence characteristics after cardiac arrest and sham arrest. Coherence between EPN spikes and MCx LFP (top row), EPN spikes and EPN LFP (middle row), and EPN LFP and MCX LFP (bottom row) are shown with SE (vertical error bars). The left-hand column shows coherence spectra across $0-100 \mathrm{~Hz}$. The $95 \%$ confidence interval for coherence averaged across all frequencies is shown for EPN spike coherence with MCX LFP in rats after cardiac arrest (shaded box, 95\% Cl: $0.026-0.90$ ). The right-hand column shows the average coherence across different frequency bands. The significant coherence level of 0.03 between spike and LFP recordings is indicated by horizontal dashed lines $(P<0.05)$. Asterisks indicate significant differences in coherence between cardiac arrest (black) and sham arrest (gray): ${ }^{* *}$ ANOVA to compare spectra, ${ }^{*}$ Student's $t$-test with Bonferroni correction to compare average power, $P<0.05$. EPN, entopeduncular nucleus; LFP, local field potentials; MCx, motor cortex.

and cardiac arrest in both the raw spectra and when normalized to total power across the $0-100 \mathrm{~Hz}$ range (Figure 3).

\section{Evaluation of Motor Function}

Immediately after cardiac arrest and resuscitation, rats demonstrate stiff extension of lower extremities (Figure 4a). For the next 5-7 d, rats continue to demonstrate motor deficits with stiff extension of the lower extremities, impaired locomotion, and increased hindlimb clasping with tail suspension (Figure 4b). Motor deficits become more subtle 7-9 mo later. Chronically, the number of rearing events during a 10-min interval was not statistically different between cardiac arrest (39 events) and sham rats (42 events; Mann-Whitney $U$-test $z=-0.76 ; P=0.22)$. Evaluation of more complex motor function using a modified Metz ladder rung test (20), however, reveals persistent deficits. Cardiac arrest rats made more hindlimb (1.2 vs. 0.6 ), forelimb (2.6 vs. 1.9 ), and total errors (3.8 vs. 2.5 ) than sham rats (Figure 5 ; Mann-Whitney $U$-test $z=1.77 ; P=0.038)$.

\section{DISCUSSION}

These results are the first to show chronic electrophysiologic changes in the neural network governing movement after cardiac arrest. Decreased EPN firing rates and increased coherence between EPN spike activity and MCx LFP may contribute to the movement disorders observed in cardiac arrest survivors.

\section{Decreased EPN Firing Rate May Reflect Decreased Excitatory Input From the STN After Cardiac Arrest}

Decreased EPN firing rates are suggestive of either increased inhibitory input or decreased excitatory input to the EPN. Like the primate GPi, the EPN receives inhibitory GABAergic input from the caudate, putamen, and the globus pallidus pars externa and receives excitatory glutamatergic input from the STN (21). The putamen, globus pallidus pars externa, and STN all exhibit neuronal loss after hypoxic-ischemic brain injury $(4,5,22)$. As damage of the putamen and globus pallidus pars externa would result in decreased inhibitory input to the EPN (and consequently increased EPN firing rates), these results support decreased excitatory input from a damaged STN as the predominant mediator of EPN firing rates after asphyxial cardiac arrest. Noting that the STN also sends sparse projections to the MCx (23-25) and that LFPs largely reflect synaptic input activity (26), disruption of shared excitatory STN input to the EPN and MCX could also contribute to the decreased coherence between EPN LFP and MCx LFP observed after cardiac arrest.

STN damage may be more associated with dystonic movement disorders post-arrest (27) as opposed to other postarrest movement disorder phenotypes like parkinsonism (4). Decreased GPi firing rates have also been shown in dystonia (12) while GPi firing rates are typically unaffected with parkinsonism $(12,16,28)$. Furthermore, in accordance with the 


\section{Articles | Aravamuthan and Shoykhet}
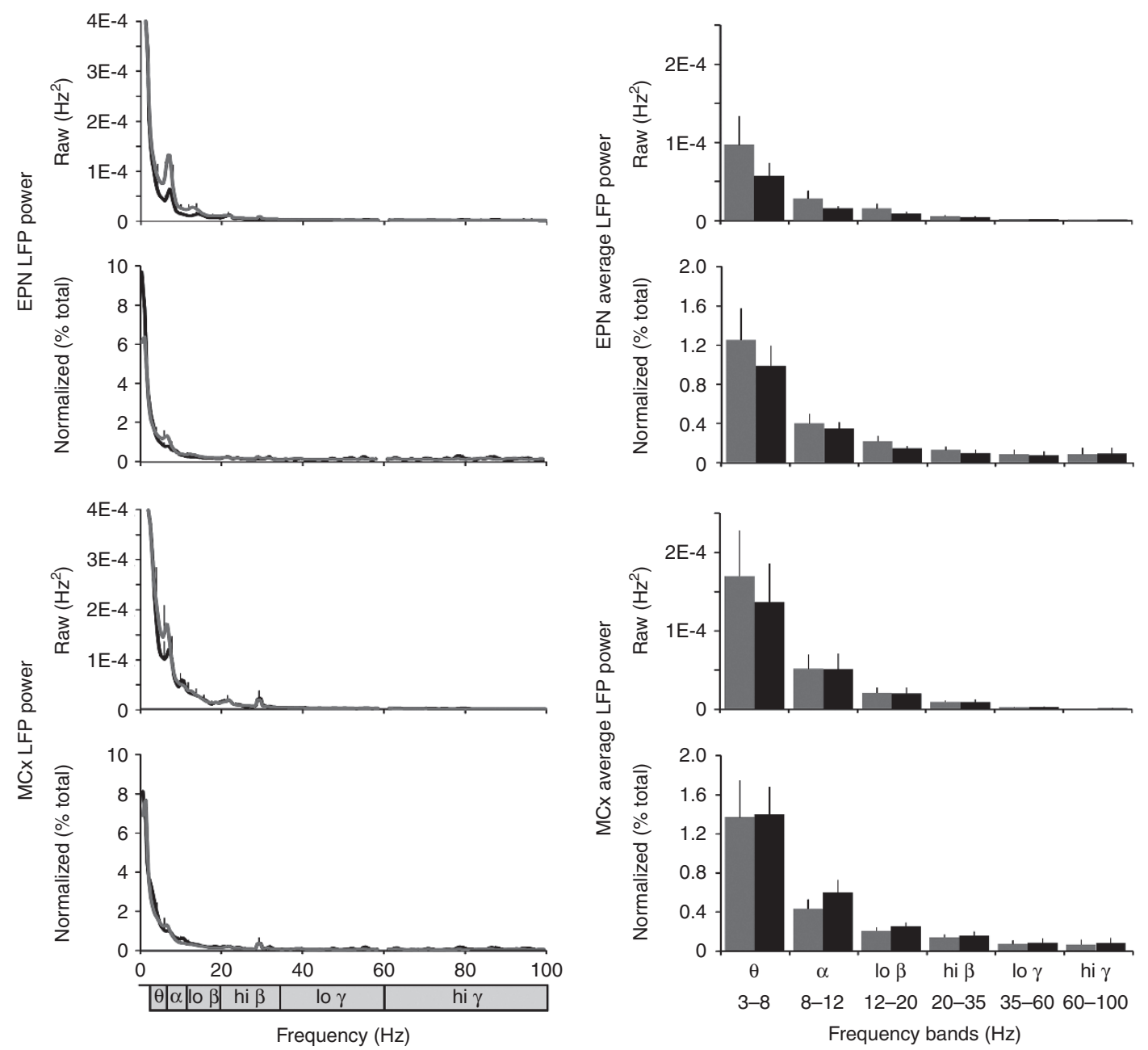

Figure 3. EPN and MCX LFP characteristics after cardiac arrest and sham arrest. EPN LFP power (top two rows) and MCx LFP power (bottom two rows) are shown with SE (vertical error bar). Power and coherence spectra across $0-100 \mathrm{~Hz}$ (left) and average power and coherence across different frequency bands (right) are shown. Raw power (first and third rows) was normalized (second and fourth rows) by dividing power values by total power across the $0-100 \mathrm{~Hz}$ frequency range. The significant coherence level of 0.03 between LFP recordings is indicated by horizontal dashed lines $(P<0.05)$. There are no significant differences in power or coherence between rats after cardiac arrest (black) vs. sham arrest (gray). EPN, entopeduncular nucleus; LFP, local field potentials; MCx, motor cortex.
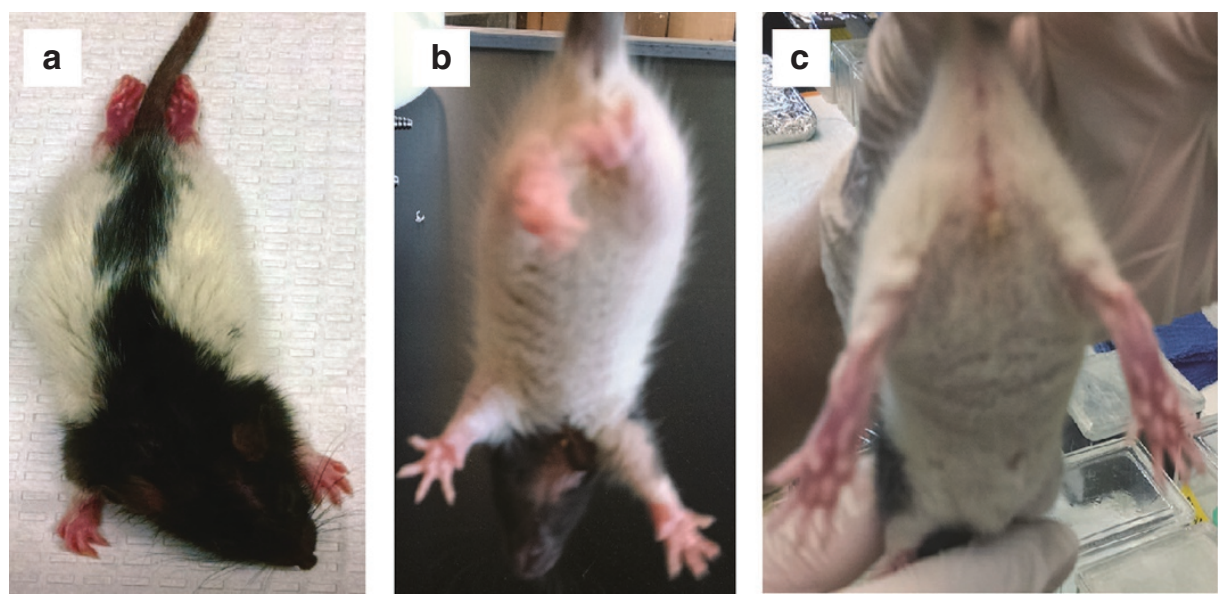

Figure 4. Short-term motor deficits in immature rats after cardiac arrest and resuscitation. (a) Four hours after cardiac arrest and resuscitation, the hindlimbs are held in stiff extension. (b) One day later, the hindlimbs still demonstrate increased tone. (c) Sham rat $1 \mathrm{~d}$ after sham intervention. 

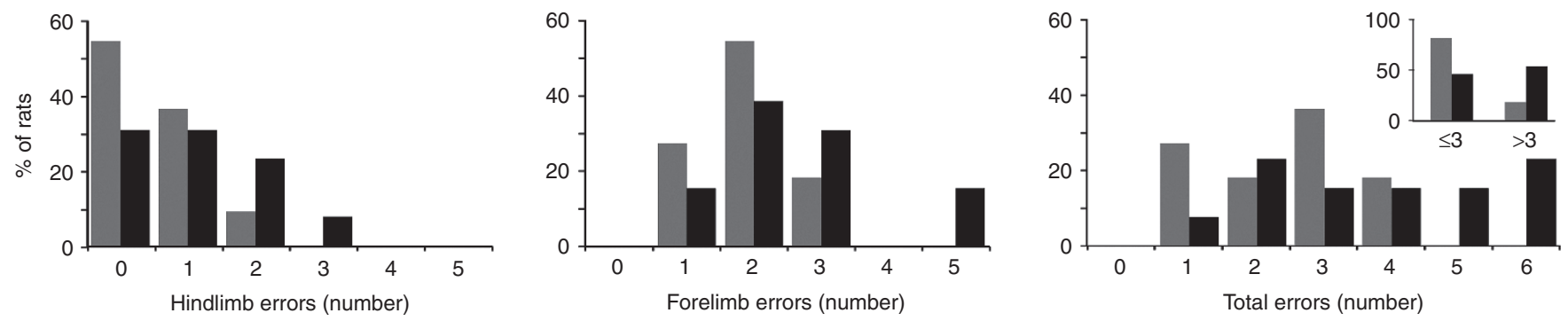

Figure 5. Long-term motor deficits in the Metz ladder rung test after cardiac arrest and resuscitation. Distribution of the number of hindlimb errors (left), forelimb errors (middle), and total errors (right, far right inset) in rats after cardiac arrest (black) vs. sham arrest (gray).

low coherence between EPN spike and EPN LFP shown in rats after cardiac arrest, only $10 \%$ of GPi neurons are coherent with GPi LFP in dystonic patients (12). Therefore, decreased EPN firing rates and absence of EPN spike coherence with EPN LFP in the rodent asphyxial cardiac arrest model may correlate with motor network changes associated with post-arrest dystonia.

\section{Synchrony Between Structures Within the Basal Ganglia- Thalamo-Cortical Motor Network Increases After Cardiac Arrest} Increased EPN spike coherence with MCx LFP was observed chronically after cardiac arrest without increased coherence between EPN spike and EPN LFP (Figure 2). Increased EPN spike coherence with MCx LFP could be due to higher fidelity transmission of EPN output to the MCx. Given that LFP primarily reflects synaptic input activity (26), another possibility is that increased EPN spike coherence with MCx LFP could reflect increased shared input between these structures after cardiac arrest. However, increased shared input between the MCx and EPN yielding increased EPN spike coherence with MCx LFP should also result in increased coherence between EPN spike and EPN LFP after cardiac arrest, which was not observed. Furthermore, decreased coherence between MCX LFP and EPN LFP after cardiac arrest is more consistent with decreased shared input between these structures. Therefore, increased EPN spike coherence with MCx LFP is most suggestive of increased transmission of basal ganglia output to the MCx after cardiac arrest.

EPN output reaches the MCx via the ventrolateral thalamic nucleus $(29,30)$ with thalamic relay modulated by the inhibitory thalamic reticular nucleus $(15,31)$. After cardiac arrest, loss or dysfunction of inhibitory neurons in the reticular nucleus (14) may result in pathologically robust thalamic transmission of EPN output to the MCx.

Increased synchrony within the basal ganglia-thalamo-cortical motor network also occurs with loss of dopaminergic modulatory gating of cortical input to the striatum $(7,8)$. Transient striatal increases in extracellular dopamine followed chronically by loss of striatal dopaminergic D2 receptor expression occurs after hypoxic-ischemic encephalopathy and in animal models of cerebral ischemia and cardiac arrest $(32,33)$. This alteration of striatal dopamine signaling may potentiate basal ganglia-thalamo-cortical motor network synchrony after cardiac arrest. However, loss of dopaminergic modulatory gating is unlikely to be the primary mediator of motor network pathology after arrest since our data indicates increased transmission of basal ganglia output to the $\mathrm{MCx}$ via the thalamus. Diminished dopaminergic modulatory gating would instead manifest as increased transmission of MCx output to the basal ganglia via the striatum.

Increased EPN spike synchrony with MCx LFP was particularly evident in a narrow $\beta$ frequency range $(20-22 \mathrm{~Hz}$ ) after arrest (Figure 2). Beta activity is more characteristic of the parkinsonian $\mathrm{GPi}(7,8)$ while $\theta$ band oscillatory activity predominates in the dystonic GPi (9-12). However, oscillatory activity in both of these frequency bands, especially in the low $\beta$ frequency range of $12-20 \mathrm{~Hz}$, is present and thought to be antikinetic in both Parkinson's disease and dystonia $(7,8,11,12)$.

Motor network synchrony observed in primary movement disorders and motor network synchrony observed chronically after cardiac arrest differ primarily in the range of affected frequencies. In primary movement disorders, increased synchrony is almost exclusively observed in the $\theta, \alpha$, and $\beta$ frequency bands. In adult rodent survivors of cardiac arrest, increased synchrony is observed across all frequency bands $(3-100 \mathrm{~Hz})$ which includes the classically prokinetic $\gamma$ band. This may be reflective of a different mechanism of basal ganglia-thalamo-cortical synchrony: increased synchrony in primary movement disorders is thought to be due to a loss of dopaminergic modulatory gating in the striatum while motor network synchrony after cardiac arrest may be more due to loss of inhibitory GABAergic modulatory gaiting in the thalamus. Increased synchrony across all frequency bands, including the prokinetic $\gamma$ band, may also be due to progressive motor recovery in these animals. Recording in these animals more proximal to the time of cardiac arrest, when they appear to show globally diminished movement, may show different frequency band patterns of motor network synchrony.

\section{Clinical Correlates of Movement Abnormalities Observed in Rats After Recovery From Cardiac Arrest}

Rats exhibited both acute and chronic motor impairments after recovery from cardiac arrest. Acutely, hindlimb stiff extension could be a corollary of the hindlimb predominant spasticity observed in patients after recovery from hypoxic-ischemic brain injury (3-6). Chronically, diminished ability to perform more complex ambulatory motor tasks could be suggestive of persistent milder appendicular spasticity, dystonia, ataxia, or a failure to adapt and execute motor plans in a novel setting. 
Intact general locomotive drive, as evidenced by unchanged rearing frequency, makes it less likely that diminished motor volition was a primary contributor to motor errors. Ultimately, presence of a clear movement disorder chronically in rats after recovery from cardiac arrest is reassuring that the electrophysiological changes observed in the EPN and MCx could truly reflect motor pathology.

\section{Functional Implications for Patients With Hypoxic-Ischemic Brain Injury}

These results suggest that motor network electrophysiologic changes may contribute to movement disorders seen after cardiac arrest. This may help guide clinical management of posthypoxic movement disorders. For example, deep brain stimulation of the GPi results in dystonic motor improvement in cerebral palsy patients $(34,35)$. GPi deep brain stimulation may be effective in cerebral palsy patients by suppressing pathologic $\beta$ oscillatory activity, as has been demonstrated in patients with primary dystonia (36) and Parkinson's disease (37).

Future research can examine whether sensory stimulation or passive movement during recording results in modulation of different oscillatory frequencies in the EPN and MCx. Electrophysiologic changes in the EPN and MCx could also be correlated with degree of functional impairment after cardiac arrest. Finally, simultaneous recording from the GPi and $\mathrm{MCx}$ intraoperatively in cerebral palsy patients undergoing deep brain stimulation electrode implantation for dystonia may help corroborate these results.

\section{METHODS}

\section{Animals}

All animal procedures were approved by the Institutional Animal Care and Use Committee at Washington University in St. Louis. Long Evans rats (Harlan Laboratories, Indianapolis, IN) were used for all experiments and were housed with their mothers until P30. Day of birth was designated as P1. For neurophysiologic experiments, the rats underwent either cardiac arrest $(n=9)$ or sham $(n=8)$ intervention at P17-19. The experimenter was blind to the injury status of the rats at the time of the recordings, motor function evaluation, and data analyses.

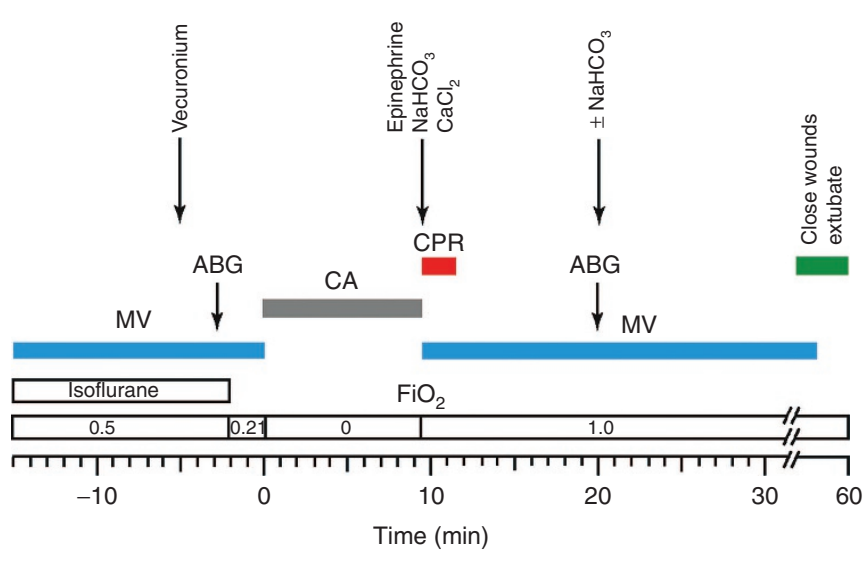

Figure 6. Schematic of the asphyxial cardiac arrest model in developing rats. $A B G$, arterial blood gas; $C A$, cardiac arrest; $C P R$, cardiopulmonary resuscitation; $\mathrm{FiO}_{2}$, fraction of inspired oxygen; $\mathrm{MV}$, mechanical ventilation.

\section{Cardiac Arrest and Resuscitation}

The model of developmental asphyxial cardiac arrest (Figure 6) has been described in detail previously (14) and was chosen to model pediatric cardiac arrest, the majority of which is respiratory in etiology $(1,2)$. Briefly, P17-19 rats underwent tracheal intubation and placement of femoral arterial and venous lines under isoflurane anesthesia. The rats were mechanically ventilated under neuromuscular blockade. Arterial blood pressure, electroencephalogram, and electrocardiogram were continuously monitored. Core body temperature was maintained at $37^{\circ} \mathrm{C}$ with a servo-controlled heating blanket. Two minutes prior to asphyxia, isoflurane was discontinued, and the animals were ventilated with room air. Such anesthetic washout is necessary to minimize the confounding effect of anesthesia on injury and recovery (38). At the end of $2 \mathrm{~min}$, the animals are just beginning to emerge from anesthesia based on EEG patterns but have yet to recover consciousness (14). After anesthetic washout, rats were disconnected from the ventilator for $9.5 \mathrm{~min}$. Pulseless electrical activity, as demonstrated by EKG and the arterial pressure waveform, ensues within 60-90 s of apnea. After $9.5 \mathrm{~min}$ of cardiac arrest, the rat was resuscitated with mechanical ventilation using $100 \% \mathrm{O}_{2}$, intravenous epinephrine $(0.01 \mathrm{mg} / \mathrm{kg}), \mathrm{NaHCO}_{3}$ (2 mEq/kg), $\mathrm{CaCl}_{2}(10 \mathrm{mg} / \mathrm{kg})$, and manual chest compressions $(\approx 300 /$ min). Upon return of spontaneous circulation, the rats additionally received a $20 \mathrm{ml} / \mathrm{kg}$ bolus of $5 \%$ dextrose in $0.9 \% \mathrm{NaCl}$ intravenously to prevent dehydration. A post-arrest arterial blood gas was obtained $10 \mathrm{~min}$ after return of spontaneous circulation, and an additional dose of $\mathrm{NaHCO}_{3}$ was given for a metabolic acidosis with $\mathrm{pH}<7.3$. Arterial and venous lines were then removed, all wounds were closed, and upon return of spontaneous respiratory effort $(\approx 1 \mathrm{~h})$, mechanical ventilation was discontinued. The rats were observed for an additional $1 \mathrm{~h}$ in a chamber with $100 \% \mathrm{O}_{2}$ and then returned to the mother. The survival rate in this model is $\approx 85 \%$. Sham intervention consisted of all anesthetic and surgical procedures except the asphyxia and resuscitation.

\section{Surgical Preparation for Electrophysiologic Recordings}

Six to nine months after cardiac arrest and resuscitation, the rat underwent tracheotomy and placement of external jugular venous and femoral arterial catheters under isoflurane anesthesia. The rat was then transferred to a stereotaxic apparatus (David Kopf Instruments, Tujunga, CA) for craniotomy, ground screw placement, and electrode placement. Craniotomies $\left(\approx 2 \mathrm{~mm}^{2}\right)$ were performed through the skull overlying the $\mathrm{MCx}, 1.5 \mathrm{~mm}$ rostral to bregma and $3.0 \mathrm{~mm}$ lateral to midline, and the EPN, $2.3 \mathrm{~mm}$ caudal to bregma and $3.0 \mathrm{~mm}$ lateral to midline (39). Dura mater was left intact and was bathed with normal saline throughout the recording. A ground screw was placed through the skull and fixed with dental acrylic. Mechanical ventilation using a volume-controlled Inspira ventilator (Harvard Apparatus, Holliston, MA) was initiated using tidal volumes of $\approx 8 \mathrm{ml} / \mathrm{kg}$ and rates of 60 breaths $/ \mathrm{min}$. Neuromuscular blockade was initiated with a bolus dose of vecuronium bromide $(1.5 \mathrm{mg} /$ $\mathrm{kg}$ ) and maintained with a continuous infusion of vecuronium (1 $\left.\mathrm{mg} \cdot \mathrm{kg}^{-1} \cdot \mathrm{h}^{-1}\right)$ in $5 \%$ dextrose $/ 0.9 \% \mathrm{NaCl}$ for the remainder of the experiment. The rat was transitioned from isoflurane to fentanyl analgesia using continuous fentanyl infusion at $10 \mu \mathrm{g} \cdot \mathrm{kg}^{-1} \cdot \mathrm{h}^{-1}$. At these doses, the rats enter a state of dissociative analgesia without compromise of thalamocortical network dynamics observed under anesthesia (40).

The rat's physiologic state during the recording session was continuously monitored as described previously (14). Briefly, temperature was maintained at $37^{\circ} \mathrm{C}$ using a servo-controlled heating blanket (Harvard Apparatus). Intra-arterial pressure and heart rate were monitored using a blood pressure monitor (WPI, Sarasota, FL) connected to the arterial line via a pressure transducer. If mean arterial pressure did not remain in the appropriate range, experiments were discontinued. Adequate analgesia was assured throughout the recording session by lack of mean arterial pressure and heart rate elevation in response to gentle touch. At the end of the recording session, the rats were killed and perfused transcardially.

\section{Electrophysiologic Recordings}

Extracellular recordings were obtained using stainless steel microelectrodes (6-8 $\mathrm{M} \Omega$ impedance at $1 \mathrm{kHz}$; FHC, Bowdoin, ME). For MCx LFP recordings, an electrode was advanced at $20^{\circ}$ from the perpendicular to a depth of $1.0 \mathrm{~mm}$ using the stereotaxic arm to target MCx layer 
$\mathrm{V}$ (which receives input from basal ganglia via the ventral lateral thalamic nucleus). For EPN recordings, the electrode was advanced perpendicularly to the pia using a hydraulic micropositioner (David Kopf Instruments). The EPN was localized relative to the ventral posteromedial and ventral posterolateral nuclei. Both in sham animals and in animals after cardiac arrest, entry into ventral posteromedial and ventral posterolateral was signaled by an abrupt increase in spontaneous and evoked neural activity, which abruptly decreased after exit from these structures. Traversing the internal capsule over a $500 \mu \mathrm{m}$ range inferior to the ventral posterolateral margin was associated with relative electrophysiological silence. Entry into the EPN was then signaled by abrupt increase in neuronal activity. Exit past the inferior margin of the EPN into the inferior portion of the internal capsule was again signaled by relative electrophysiological silence (39). The raw wide-band signal was passed through a headstage at $1 \times$ amplification, digitized at $40 \mathrm{kHz} /$ channel with Omniplex DigiAmp (Plexon Inc, Dallas, TX) and then digitally filtered between $300 \mathrm{~Hz}$ and $10 \mathrm{kHz}$ for spike trains and between 1 and $300 \mathrm{~Hz}$ for LFP recordings. Wide-band, LFP, and spiketrain signals were stored in .plx format for further analyses.

\section{Electrophysiologic Data Analysis}

Five to ten minutes of baseline activity were recorded simultaneously from the MCx and the EPN for each isolated EPN single unit. From this recording, continuous $300 \mathrm{~s}$ of data free from artifacts were
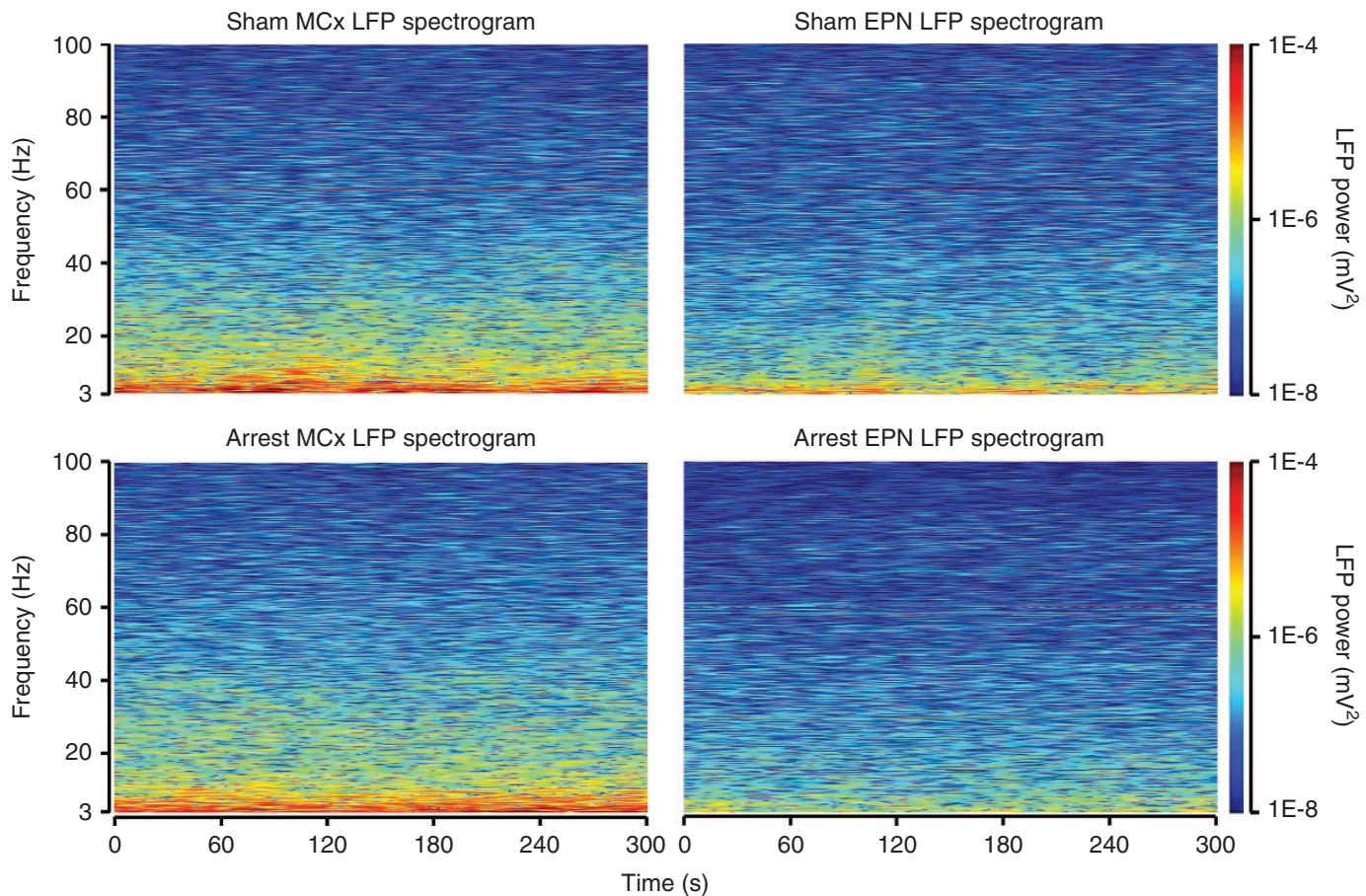

Figure 7. Example spectrograms of simultaneously recorded MCx (left) and EPN (right) LFPs in rats after sham arrest (top row) and cardiac arrest (bottom row). LFP power between 3 and $100 \mathrm{~Hz}$ remained stable over the 300-s period used for data analysis in both structures in all recordings. LFP, local field potentials; MCx, motor cortex.

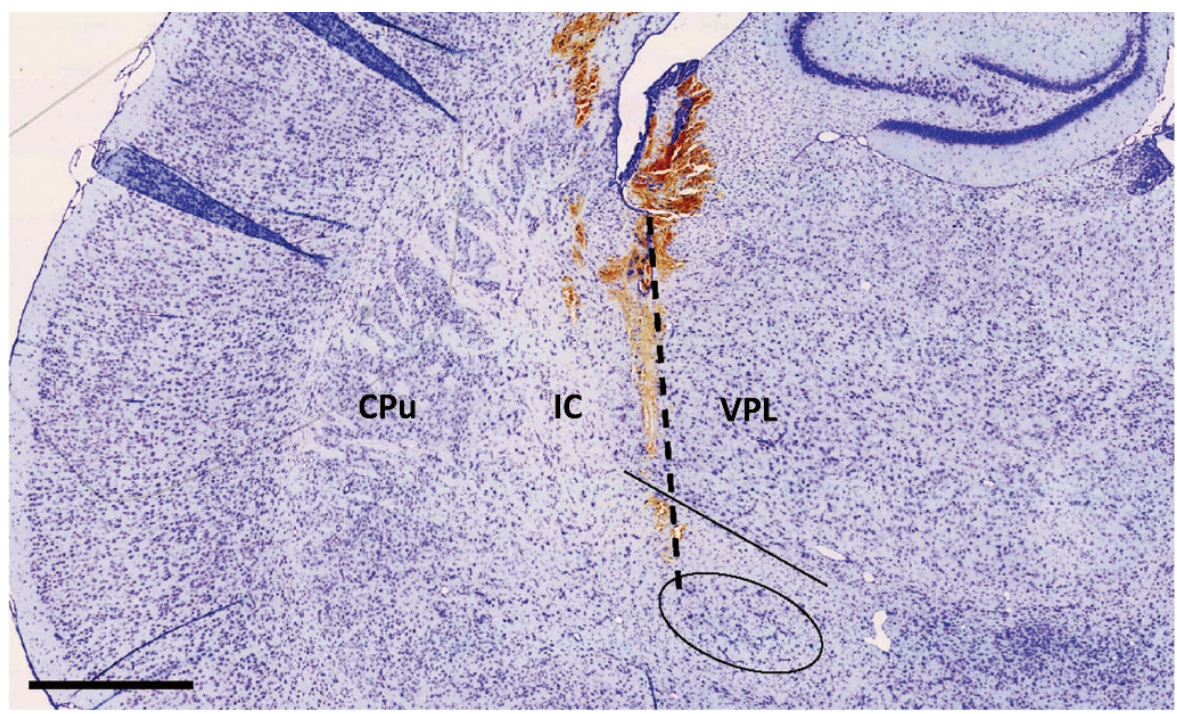

Figure 8. Localization of the EPN on a cresyl violet-stained $10 \mu \mathrm{m}$ rat brain slice. The electrode tract (dashed line) can be clearly visualized passing through the EPN (oval) in this slice $2.5 \mathrm{~mm}$ posterior to bregma (39). The straight line shows the border of inferior border of the thalamic reticular nucleus. Bar $=1 \mathrm{~mm}$. CPu, caudate/putamen; EPN, entopeduncular nucleus; IC, internal capsule; VPL, ventroposterior medial nucleus. 
selected. Single units were isolated from EPN recordings using the Plexon Offline Sorter (Plexon) by direct visualization of 3D waveform clusters in principal component space. All single units had interspike intervals $>1 \mathrm{~ms}$. At most, two single units were recorded simultaneously on a single electrode in EPN.

NeuroExplorer 4.0 (Nex Technologies, Madison, AL) was used for firing rate, power, and coherence data analyses. Firing rate for each cell was calculated as the average firing rate across the 300 s recording period. Fast Fourier transform (FFT) analysis was used to determine the power spectral density of spike trains (calculated using the rate histogram for each window, de-trended by subtraction of its linear regression) and LFPs for each $300 \mathrm{~s}$ data segment using a block size of 512 across the $0-100 \mathrm{~Hz}$ frequency range. Power spectral density plots of simultaneously recorded LFPs from the MCx and EPN were examined over the $300 \mathrm{~s}$ recording duration to ensure that LFP power spectra were stable across all frequencies over time, indicating stable states of motor volition during the recording (Figure 7) $(7,13)$.

Coherence between neuronal spiking activity and LFP activity across the $0-100 \mathrm{~Hz}$ frequency range was calculated as follows: (i) each $300 \mathrm{~s}$ data segment was divided into $5 \mathrm{~ms}$ bins; (ii) 117 nonoverlapping blocks consisting of consecutive 512 bins were allocated (2.56 $\mathrm{s}$ windows); (iii) FFTs and the complex conjugate (Conj) of the FFTs were calculated for each block; (iv) individual and cross-densities were calculated between the reference MCx LFP signal (X) and the target EPN LFP or spike activity signal (Y):

$$
\begin{aligned}
& \mathrm{P}_{\mathrm{XX}}=\mathrm{FFT}(\mathrm{X})^{\star} \operatorname{Conj}(\mathrm{FFT}(\mathrm{X})) \\
& \mathrm{P}_{\mathrm{YY}}=\mathrm{FFT}(\mathrm{Y})^{\star} \operatorname{Conj}(\mathrm{FFT}(\mathrm{Y})) \\
& \mathrm{P}_{\mathrm{XY}}=\mathrm{FFT}(\mathrm{X})^{\star} \operatorname{Conj}(\mathrm{FFT}(\mathrm{Y}))
\end{aligned}
$$

(v) Pxx, Pyy, and Pxy values were averaged across all blocks. Coherence values were calculated as:

$$
\mathrm{C}=\left(\operatorname{Mean}\left(\mathrm{P}_{\mathrm{XY}}\right)\right)^{2} /\left(\operatorname{Mean}\left(\mathrm{P}_{\mathrm{XX}}\right)^{\star} \operatorname{Mean}\left(\mathrm{P}_{\mathrm{YY}}\right)\right)
$$

Coherence statistical significance was determined from the following equation:

$$
\mathrm{C}_{\text {crit }}=1-(1-\alpha)^{1 /(\mathrm{L}-1)}
$$

where $\alpha$ is the confidence limit and L is the number of windows. For this study, $\alpha=0.95$ and $\mathrm{L}=117$. Hence, $\mathrm{C}_{\text {crit }}=0.03$, and coherence $>\mathrm{C}_{\text {crit }}$ was considered statistically significant. Coherence between LFP signals in EPN and MCx was calculated similarly. In addition to examining power and coherence spectra across the $0-100 \mathrm{~Hz}$ range, average power and coherence was also determined for each of six functionally significant frequency bands: $\theta(3-8 \mathrm{~Hz}), \alpha(8-12 \mathrm{~Hz})$, low $\beta$ (lo $\beta, 12-20 \mathrm{~Hz}$ ), high $\beta$ (hi $\beta, 20-35 \mathrm{~Hz}$ ), low $\gamma$ (lo $\gamma, 35-60$ $\mathrm{Hz}$ ), and high $\gamma($ hi $\gamma, 60-100 \mathrm{~Hz}$ ). Firing rate, spike power, and spiketo-LFP coherence were averaged across all cells. LFP power and LFPto-LFP coherence were averaged across rats.

Data are presented as the mean \pm SEM and were statistically evaluated with grouped $t$-test or ANOVA. Criterion of significance was $P$ $<0.05$.

\section{Histology}

Rats used in neurophysiologic recordings were perfused with $4 \%$ paraformaldehyde, their brains were extracted, postfixed for $24-48 \mathrm{~h}$ at $4 \%$ paraformaldehyde and then for an additional 12 to $14 \mathrm{~h}$ in $70 \%$ ethanol. Brains were embedded in paraffin, sliced into $10 \mu \mathrm{m}$ sections, and stained with cresyl violet. Intraoperative localization of recording electrodes in EPN was confirmed postmortem by direct visualization of the electrode tracks (Figure 8).

\section{Assessment of Motor Function}

A separate cohort of 7-9-mo-old rats that underwent either a sham intervention $(n=11)$ or asphyxial cardiac arrest during the third postnatal week $(n=13)$ was tested on a modified Metz ladder rung test (20). The ladder rung apparatus consisted of two clear polycarbonate walls $1 \mathrm{~m}$ in length and $0.2 \mathrm{~m}$ in height with metal rungs $3 \mathrm{~mm}$ in diameter placed at $2 \mathrm{~cm}$ intervals at the bottom of the walls. Rats were allowed one practice run followed by a test run. Each rat was tested once to minimize the effect of pattern learning on motor performance. Rats were recorded with a digital camera (Panasonic HDC-TM80; Panasonic, Osaka, Japan), and performance was analyzed offline. The errors were scored when the rat completely missed the rung resulting in a fall or when one of the paws slipped off the rung resulting in a fall. Only the first paw to fail in a gait cycle was scored as an error. Scoring resumed once all paws were repositioned on the rungs. Using the same cohort, we additionally analyzed general locomotion by measuring the number of rearing events during a 10 -min period when rats were placed in clear-walled plastic box $(0.5 \times 0.5 \times 0.75 \mathrm{~m})$. All testing was conducted during the day. Results of both the Metz ladder rung test and the rearing test were analyzed using the Mann-Whitney $U$-test with the null hypothesis that cardiac arrest rats perform as well as sham rats on each task.

\section{STATEMENT OF FINANCIAL SUPPORT}

This study was supported by National Institute of Neurological Disorders and Stroke, Bethesda, MD, USA (K08 NS-082362); The Children's Discovery Institute, St. Louis, MO, USA; Child Health Research Center, St. Louis, MO, USA (K12-HD076224); McDonnell Center for Systems Neuroscience at Washington University in St. Louis, St. Louis, MO, USA. B.R.A. was supported by St. Louis Children's Hospital, St. Louis, MO, USA.

Disclosures: The authors have no disclosures, financial ties to products in the study, or conflicts of interest.

\section{REFERENCES}

1. Donoghue AJ, Nadkarni V, Berg RA, et al.; CanAm Pediatric Cardiac Arrest Investigators. Out-of-hospital pediatric cardiac arrest: an epidemiologic review and assessment of current knowledge. Ann Emerg Med 2005;46:512-22.

2. Neumar RW, Nolan JP, Adrie C, et al. Post-cardiac arrest syndrome: epidemiology, pathophysiology, treatment, and prognostication. A consensus statement from the International Liaison Committee on Resuscitation (American Heart Association, Australian and New Zealand Council on Resuscitation, European Resuscitation Council, Heart and Stroke Foundation of Canada, InterAmerican Heart Foundation, Resuscitation Council of Asia, and the Resuscitation Council of Southern Africa); the American Heart Association Emergency Cardiovascular Care Committee; the Council on Cardiovascular Surgery and Anesthesia; the Council on Cardiopulmonary, Perioperative, and Critical Care; the Council on Clinical Cardiology; and the Stroke Council. Circulation 2008;118:2452-83.

3. Martinez-Biarge M, Diez-Sebastian J, Kapellou O, et al. Predicting motor outcome and death in term hypoxic-ischemic encephalopathy. Neurology 2011;76:2055-61.

4. Khot S, Tirschwell DL. Long-term neurological complications after hypoxic-ischemic encephalopathy. Semin Neurol 2006;26:422-31.

5. Hawker K, Lang AE. Hypoxic-ischemic damage of the basal ganglia. Case reports and a review of the literature. Mov Disord 1990;5:219-24.

6. Venkatesan A, Frucht S. Movement disorders after resuscitation from cardiac arrest. Neurol Clin 2006;24:123-32.

7. Brown P. Abnormal oscillatory synchronisation in the motor system leads to impaired movement. Curr Opin Neurobiol 2007;17:656-64.

8. Hutchison WD, Dostrovsky JO, Walters JR, et al. Neuronal oscillations in the basal ganglia and movement disorders: evidence from whole animal and human recordings. J Neurosci 2004;24:9240-3.

9. Chen CC, Brown P. The role of synchronised low frequency activity in globus pallidus interna in dystonia. Acta Neurol Taiwan 2007;16:1-6.

10. Chen CC, Kühn AA, Trottenberg T, Kupsch A, Schneider GH, Brown P. Neuronal activity in globus pallidus interna can be synchronized to local field potential activity over $3-12 \mathrm{~Hz}$ in patients with dystonia. Exp Neurol 2006;202:480-6.

11. Liu X, Wang S, Yianni J, et al. The sensory and motor representation of synchronized oscillations in the globus pallidus in patients with primary dystonia. Brain 2008;131(Pt 6):1562-73.

12. Weinberger M, Hutchison WD, Alavi M, et al. Oscillatory activity in the globus pallidus internus: comparison between Parkinson's disease and dystonia. Clin Neurophysiol 2012;123:358-68.

13. Jenkinson N, Kühn AA, Brown P. $\gamma$ oscillations in the human basal ganglia. Exp Neurol 2013;245:72-6. 
14. Shoykhet M, Simons DJ, Alexander H, Hosler C, Kochanek PM, Clark RS. Thalamocortical dysfunction and thalamic injury after asphyxial cardiac arrest in developing rats. J Neurosci 2012;32:4972-81.

15. Humphries MD, Gurney KN. The role of intra-thalamic and thalamocortical circuits in action selection. Network 2002;13:131-56.

16. Ruskin DN, Bergstrom DA, Walters JR. Nigrostriatal lesion and dopamine agonists affect firing patterns of rodent entopeduncular nucleus neurons. J Neurophysiol 2002;88:487-96.

17. Benhamou L, Cohen D. Electrophysiological characterization of entopeduncular nucleus neurons in anesthetized and freely moving rats. Front Syst Neurosci 2014;8:7.

18. Nini A, Feingold A, Slovin H, Bergman H. Neurons in the globus pallidus do not show correlated activity in the normal monkey, but phase-locked oscillations appear in the MPTP model of parkinsonism. J Neurophysiol 1995;74:1800-5.

19. Raz A, Vaadia E, Bergman H. Firing patterns and correlations of spontaneous discharge of pallidal neurons in the normal and the tremulous 1-methyl-4-phenyl-1,2,3,6-tetrahydropyridine vervet model of parkinsonism. J Neurosci 2000;20:8559-71.

20. Metz GA, Whishaw IQ. The ladder rung walking task: a scoring system and its practical application. J Vis Exp 2009;28.

21. Hazrati LN, Parent A. Differential patterns of arborization of striatal and subthalamic fibers in the two pallidal segments in primates. Brain Res 1992;598:311-5.

22. Fujioka M, Okuchi K, Miyamoto S, et al. Changes in the basal ganglia and thalamus following reperfusion after complete cerebral ischaemia. Neuroradiology 1994;36:605-7.

23. Carpenter MB, Carleton SC, Keller JT, Conte P. Connections of the subthalamic nucleus in the monkey. Brain Res 1981;224:1-29.

24. Jackson A, Crossman AR. Subthalamic nucleus efferent projection to the cerebral cortex. Neuroscience 1981;6:2367-77.

25. Kita H, Kitai ST. Efferent projections of the subthalamic nucleus in the rat: light and electron microscopic analysis with the PHA-L method. J Comp Neurol 1987;260:435-52.

26. Einevoll GT, Kayser C, Logothetis NK, Panzeri S. Modelling and analysis of local field potentials for studying the function of cortical circuits. Nat Rev Neurosci 2013;14:770-85.

27. Griffiths PD, Radon MR, Crossman AR, Zurakowski D, Connolly DJ. Anatomic localization of dyskinesia in children with "profound" perinatal hypoxic-ischemic injury. AJNR Am J Neuroradiol 2010;31:436-41.
28. Tang JK, Moro E, Mahant N, et al. Neuronal firing rates and patterns in the globus pallidus internus of patients with cervical dystonia differ from those with Parkinson's disease. J Neurophysiol 2007;98:720-9.

29. Hazrati LN, Parent A. Contralateral pallidothalamic and pallidotegmental projections in primates: an anterograde and retrograde labeling study. Brain Res 1991;567:212-23.

30. Parent A, De Bellefeuille L. Organization of efferent projections from the internal segment of globus pallidus in primate as revealed by fluorescence retrograde labeling method. Brain Res 1982;245:201-13.

31. Haber SN, Calzavara R. The cortico-basal ganglia integrative network: the role of the thalamus. Brain Res Bull 2009;78:69-74.

32. Meng SZ, Isumi H, Takashima S. Neuropathological characteristics and alteration of the dopamine $\mathrm{D} 2$ receptor in hypoxic-ischemic basal ganglia necrosis. Brain Dev 1998;20:98-104.

33. Obrenovitch TP, Sarna GS, Matsumoto T, Symon L. Extracellular striatal dopamine and its metabolites during transient cerebral ischaemia. J Neurochem 1990;54:1526-32.

34. Koy A, Hellmich M, Pauls KA, et al. Effects of deep brain stimulation in dyskinetic cerebral palsy: a meta-analysis. Mov Disord 2013;28: 647-54.

35. Vidailhet M, Yelnik J, Lagrange C, et al.; French SPIDY-2 Study Group. Bilateral pallidal deep brain stimulation for the treatment of patients with dystonia-choreoathetosis cerebral palsy: a prospective pilot study. Lancet Neurol 2009;8:709-17.

36. Whitmer D, de Solages C, Hill BC, Yu H, Bronte-Stewart H. Resting beta hypersynchrony in secondary dystonia and its suppression during pallidal deep brain stimulation in DYT3+ Lubag dystonia. Neuromodulation 2013;16:200-5; discussion 205.

37. McConnell GC, So RQ, Hilliard JD, Lopomo P, Grill WM. Effective deep brain stimulation suppresses low-frequency network oscillations in the basal ganglia by regularizing neural firing patterns. J Neurosci 2012;32:15657-68.

38. Statler $\mathrm{KD}$, Alexander $\mathrm{H}$, Vagni $\mathrm{V}$, et al. Isoflurane exerts neuroprotective actions at or near the time of severe traumatic brain injury. Brain Res 2006;1076:216-24.

39. Paxinos G, Watson C. The Rat Brain in Stereotaxic Coordinates. 6th edn. Burlington, MA: Academic Press, 2007.

40. Simons DJ, Carvell GE, Hershey AE, Bryant DP. Responses of barrel cortex neurons in awake rats and effects of urethane anesthesia. Exp Brain Res 1992;91:259-72. 